\title{
BOTÁNICA
}

\section{REDESCUBRIMIENTO DE MICONIA IMBRICATA (MELASTOMATACEAE) Y SU TRANSFERENCIA AL GÉNERO KILLIPIA, Y COMENTARIOS SOBRE LA POSICIÓN DE LA INFLORESCENCIA}

\author{
Juan Mauricio Posada-Herrera*, Humberto Mendoza-Cifuentes**
}

\begin{abstract}
RESUMEN
Posada-Herrera, J.M., H. Mendoza-Cifuentes: Redescubrimiento de Miconia imbricata (Melastomataceae) y su transferencia al género Killipia, y comentarios sobre la posición de la inflorescencia. Rev. Acad. Colomb. Cienc. 37 (143): 207-213, 2013. ISSN 0370-3908.

Se redescubre Miconia imbricata Gleason, especie hasta ahora solo conocida por su colección tipo, endémica del Parque Nacional Natural Tatamá. Se completa su descripción, se evalúan las características florales y la posición de la inflorescencia y se establece su estado de conservación. El análisis morfológico revela errores de interpretación sobre la posición de la inflorescencia y una mayor afinidad de esta especie con el género Killipia, así que se procede a realizar su transferencia a este género.
\end{abstract}

Palabras Clave: Melastomataceae, Miconia, Miconieae, Killipia, Sagraea, Norte de Los Andes, Colombia, Tatamá, Taxonomía.

\begin{abstract}
Miconia imbricata Gleason, is rediscovered a species endemic of Tatamá National Park which is know only from its type. Its description is spread out, its floral characteristics and inflorescence position are evaluated and its conservation state is determined. The morphologic analysis show some mistakes from the interpretation of inflorescence position and an affinity of this species with the genus Killipia, so we proceed to conduct the transfer to this genus.
\end{abstract}

Key words: Melastomataceae, Miconia, Miconieae, Killipia, Sagraea, Noth of the Andean, Colombia, Tatamá, Taxonomy.

\section{Introducción}

El género Miconia Ruiz \& Pav. es el mayor de la familia Melastomataceae con alrededor de 1060 especies exclusi- vamente neotropicales (Goldenbreg, 2000). Colombia con cerca de 320 especies es uno de los países con mayor di-

* Calle 65 No 26 -10, Herbario Universidad de Caldas, Manizales, Caldas, Colombia, A.A. 275. Correo electronico: Juan.posada87@gmail.com

** Carrera 8 No 15-08, Claustro de San Agustín, Villa de Leiva, Boyacá, Colombia. Correo electronico: hummendoza@gmail.com 
versidad del género (Mendoza \& Ramírez, 2007). Para el país cerca del $30 \%$ de las especies de este género sólo se conocen de pocas localidades y algunas otras sólo de la colección tipo, lo que representa sin lugar a duda endemismos que ameritan su plena documentación.

En el presente artículo se documenta el redescubrimiento de M. imbricata, una especie descrita por Gleason (1925) y conocida hasta el momento sólo de la colección tipo realizada por Francis W. Pennel en 1922, en el Parque Natural Nacional de Tatamá, departamento de Risaralda. Esta especie presenta caracteres de las flores similares a los encontrados en el género Killipia Gleason, pero las inflorescencias en apariencia son terminales como ocurre en Miconia, lo que sugiere un vinculo filogenético cercano entre M. imbricata y Killipia antes no documentado. Considerando esto, se amplía la descripción de la especie, se ilustra, se establece su estado de conservación, se discute sobre la importancia filogenética de algunos caracteres florales y se transfiere M. imbricata al género Killipia.

\section{Resultados y Discusión}

\section{Relaciones filogenéticas de Killipia y la posición de la in- florescencia.}

Killipia está incluido dentro de los taxones con inflorescencias axilares en la tribu Miconieae y tradicionalmente se caracteriza por las inflorescencias axilares, flores 5-meras, lóbulos del cáliz elongados y asociados a los dientes dorsales desarrollados, anteras truncadas (oblongas), y conectivo basalmente tuberculado (Ruiz-Rodgers, 1996, 1997; Judd, 1989). Otros caracteres del género mencionados por estos autores son el tallo cuadrangular y la ausencia de cubiertas y tricomas (plantas glabras), no obstante, son variables dentro del género y no pueden ser considerados como diagnósticos. La totalidad de sus especies se encuentran a lo largo de la Cordillera Occidental en Colombia y Andes occidentales del norte de Ecuador, entre los 1500 y $2600 \mathrm{~m}$ de altitud (RuizRodgers, 1996).

Judd (1989), con base en un análisis cladístico sustentado en caracteres morfológicos, planteó la hipótesis de que Killipia se encuentra filogenéticamente más cercano a taxones de inflorescencias axilares como Sagraea, Mecranium y Pentosea, que a especies del género Miconia. Igualmente, Michelangeli et al. (2004) plantea que Killipia tienen afinidad con especies del complejo Clidemia-Sagraea de inflorescencias axilares.

Sagraea (grupo Clidemia-Sagraea), por otro lado, también se encuentra dentro del grupo de Miconieae de inflorescen- cias axilares, es considerado un grupo no natural y sus especies se asociaron a los géneros Clidemia y Ossaea (Judd, 1989). En Colombia se encuentran tres especies del este género de la Cordillera Occidental (Lozano-Contreras \& Ruiz-Rodgers, 1995) que aun no han sido transferidas a Clidemia.

La posición de la inflorescencia es un carácter más o menos conservado dentro de los géneros de la tribu Miconieae, por lo que se ha sido utilizado para delimitar sus géneros (Michelangeli et al. 2004). Sin embargo, algunos taxones tienen excepciones y este carácter no funciona bien como es el caso de Ossaea y Leandra, indicando al parecer cierta plasticidad del carácter (Michelangeli et al. 2004). No obstante, esta plasticidad puede ser un artificio ya que es posible que sea producto de la mala definición de los géneros (Michelangeli et al. 2004). El trabajo de Michelangeli et al (2004), tendiente a dilucidar la importancia filogenética de la posición de la inflorescencia, mostró que la posición lateral o terminal ha emergido varias veces de forma independiente en la tribu y que la clasificación interna con base en este carácter debe ser reexaminada pues aun no hay claridad de cuáles son los meristemas que desarrollan las inflorescencias.

La comparación de caracteres florales del grupo M. imbricata-Killipia con especies de Miconia (grupo de M. micayensis) y con el grupo Clidemia-Sagraea de la Cordillera Occidental de Colombia y montañas de Panamá, muestra que hay mayor afinidad de Killipia con Miconia (Tabla 1). Algunos caracteres de M. imbricata y Killipia que se encuentran en un grupo de especies de Miconia son la forma de la inflorescencia (corimbiforme), el tipo de corola (cucullada), el número de pétalos (5), la forma del ápice del cáliz (lóbulos membranáceos con diente dorsal ancho), y la forma del estigma (punctiforme), que no se presentan en el grupo de inflorescencias axilares de Clidemia-Sagraea (Tabla 1). Estas semejanzas en los caracteres florales permiten sugerir que el grupo killipia-M. imbricata tienen más afinidad con algunas especies del género Miconia que con Sagraea, y que la posición de la inflorescencia es un carácter variable.

Miconia imbricata es un ejemplo de que los posibles errores de interpretación de la ubicación de la inflorescencia, que es un ruido que no ha permitido dilucidar claramente las relaciones parentesco de grupos en la tribu. El grupo de Killipia, M. imbricata y las otras especies de Miconia documentadas en la Tabla 1, son un excelente conjunto para dilucidar el interrogante aun vigente del valor filogenético de la posición de la inflorescencia en la tribu Miconieae. 


\section{Notas sobre la posición de la inflorescencia en $M$. imbri- cata}

El estudio detallado de pequeños promontorios o callos remanentes en los puntos de ramificación del tallo y en la base de la inflorescencia de $M$. imbricata, permite sugerir que en esta especie la inflorescencia es axilar y no terminal como originalmente fue descrito por Gleason (1925). Los callos mencionados arriba presentan una serie de cicatrices producto de hojas y yemas caducas, que indica que corresponde a una rama o vástago supremamente reducido (menor de $5 \mathrm{~mm}$ long); en este vástago se encuentran cuatro cicatrices, dos de ellas de hojas con tres puntos o huellas de haces vasculares (que corresponden a tres venas principales de la nerviación foliar), y dos huellas sin haces vasculares evidentes; también presenta una yema apical persistente muy atrofiada pero con diminutos primordios foliares (Figura 3). De acuerdo con esto, la inflorescencia se desarrolla en la axila de una hoja en un vástago central reducido que da la apariencia de ser terminal (Figura 3).

Las características de la inflorescencia y la flor de $M$. imbricata son semejantes a las encontradas en especies del género Killipia. Entre estos caracteres se encuentran: inflorescencias cortas corimbiformes, flor 5 mera, corola cucullada, pétalos entre 4 y $8 \mathrm{~mm}$ longitud (tamaño pequeño), cáliz con lóbulos anchos membranáceos y dientes dorsales desarrollados, conectivo de la antera tuberculado en la parte basal dorsal, estigma no expandido y ovario parcialmente unido al tálamo del hipanto (Figura 1, Tabla 1). Miconia imbricata difiere de las especies de Killipia sólo en la posición de la inflorescencia, pero como se documenta anteriormente, este carácter fue

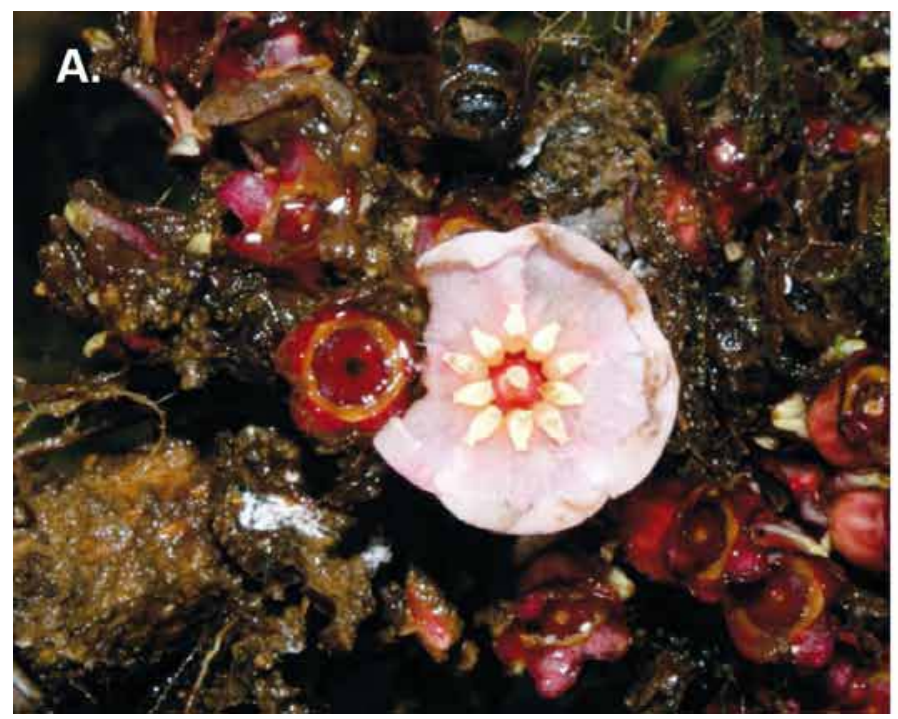

mal interpretado desde la descripción original y en verdad presenta inflorescencia axilar.

Considerando lo anterior, M. imbricata es una Killipia, así que se realiza su transferencia a este género y se amplía la descripción de esta especie con base en las anotaciones de campo, registros fotográficos y material adicional proveniente de la localidad tipo.

Killipia imbricata (Gleason) Posada-Herrera \& Humberto Mend., Comb. Nov. (Figuras 2 y 3)

Miconia imbricata Gleason, Bulletin of the Torrey Botanical Club, 52(7): 382-382 (1925). Tipo: COLOMBIA. Risaralda: Cordillera Occidental, Cerro Tatamá, 3200-3400 m, 8-10 Sep 1922, fl, F. W. Pennel 10503 (holotipo, NY).

Arbusto de 1,5-3 m; entrenudos de 1,3-1,9 cm, teretes. Ramas, peciolos e inflorescencia con cubierta densa purpura, marrones al secar, estriados; tricomas filiformes levemente adpresos de 0,4-0,8 mm. Hojas del mismo tamaño en cada nudo en ocasiones desiguales una de la otra en longitud. Peciolos teretes de 1,0-2,5 cm y 1,0-2,0 mm de diámetro. Lámina de 2-7 x 1--3 cm, ovadas, oblongovadas u oblongoelípticas, de textura cartácea quebradiza; margen denticulado-espinulosa; base obtusa a redondeada; ápice agudo o levemente acuminado; haz glabra, con el retículo de la nervadura impreso; envés con cubierta similar a los peciolos en la nervadura. Nerviación con 2 pares de venas secundarias acompañando a la vena media, basales; con 32-35 venas terciarias aledañas a la vena media y distanciadas $1,3-1,8 \mathrm{~mm}$ en la parte media de la lámina; nerviación terciaria formando

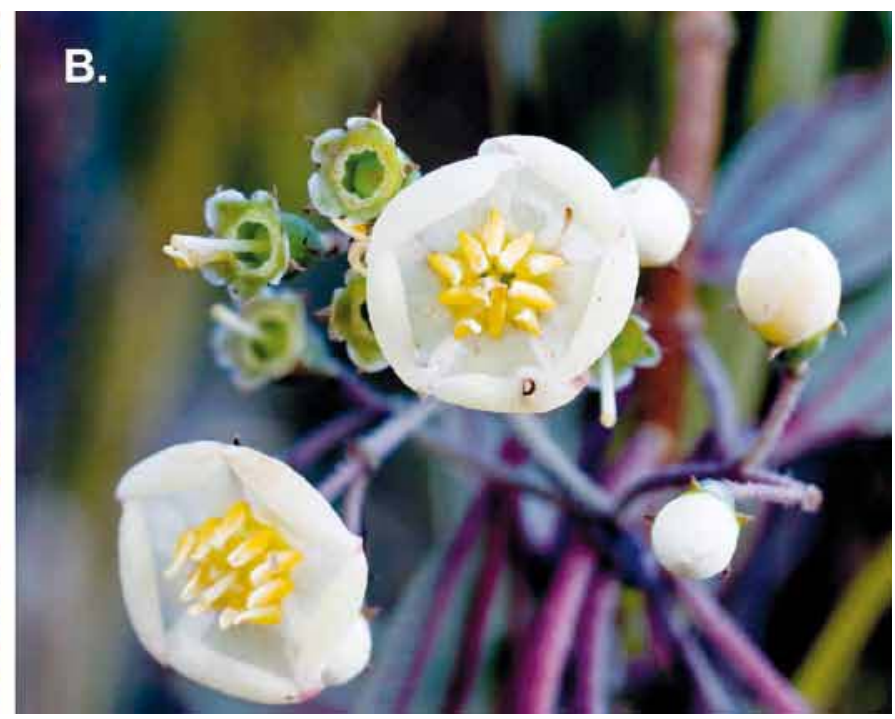

Figura 1. Comparación de flores de K. imbricata y Killipia. A. Killipia verticalis (Foto: Humberto Mendoza-Cifuentes); B. Killipia imbricata (Foto: Jasón Gutiérrez). 


\begin{tabular}{|c|c|c|c|c|c|c|c|c|c|c|c|c|c|c|c|c|c|c|c|}
\hline 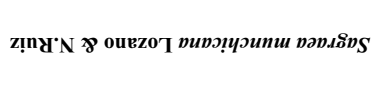 & 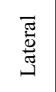 & $\begin{array}{l}\text { 总 } \\
\text { 离 }\end{array}$ & $\therefore$ & 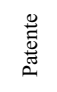 & -. & . & 离 & t & 0 & के & $z$ & $\vec{n}$ & 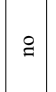 & 总: & s & 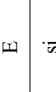 & 8 & 第导 & 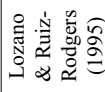 \\
\hline 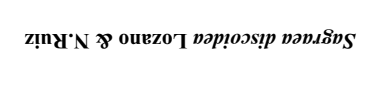 & 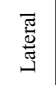 & 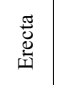 & $\therefore$ & 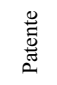 & $\therefore$ & $\frac{\stackrel{8}{0}}{0}$ & $\stackrel{\stackrel{ }{\vec{x}}}{\stackrel{\longrightarrow}{\rightarrow}}$ & t & 0 & के & $\mathrm{z}$ & $\vec{n}$ & 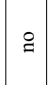 & 总: & s & 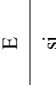 & 8 & \&్ల & 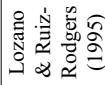 \\
\hline 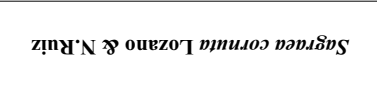 & 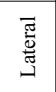 & 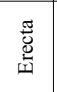 & $a$ & 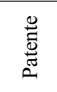 & \begin{tabular}{|l|}
$\frac{8}{0}$ \\
$\frac{0}{m}$ \\
\end{tabular} & $\frac{\stackrel{8}{0}}{\frac{\pi}{5}}$ & $\begin{array}{l}\stackrel{\infty}{0} \\
\stackrel{\dot{\alpha}}{0} \\
\stackrel{-}{+}\end{array}$ & + & 0 & की & $\bar{z}$ & " & $\cong$ & 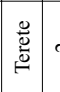 & 4 & क & 8 & 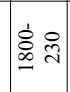 & 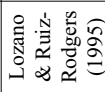 \\
\hline 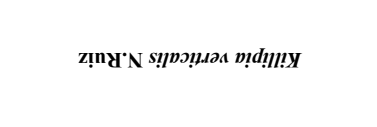 & $\frac{\sqrt{5}}{5}$ & 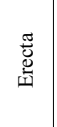 & 0 & 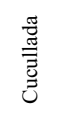 & 总 & 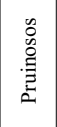 & 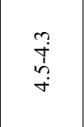 & in & $\$$ & ิิ & F & $\vec{n}$ & $\vec{n}$ & 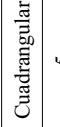 & n & 罗 & 8 & 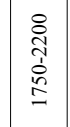 & 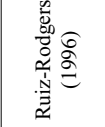 \\
\hline чэвр.п & 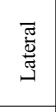 & 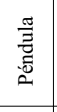 & u & 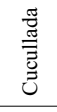 & 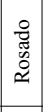 & 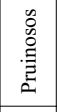 & 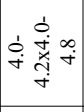 & in & $₫$ & ิิ & ค & $\vec{n}$ & $\vec{n}$ & . & m & 崖 & 8 & 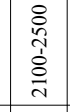 & 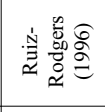 \\
\hline 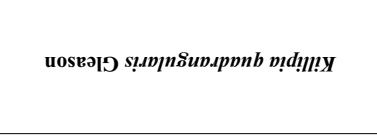 & 营 & 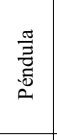 & 0 & 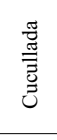 & 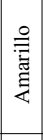 & 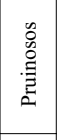 & $\begin{array}{l}\dot{1} \\
\dot{c} \\
\dot{c} \\
\dot{p} \\
\dot{b} \\
\dot{+}\end{array}$ & n & $₫$ & ิิ & f & क & $\vec{n}$ & 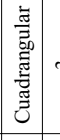 & m & \begin{tabular}{|c|c|}
$\mathbf{z}$ \\
\end{tabular} & 8 & 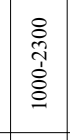 & 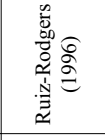 \\
\hline 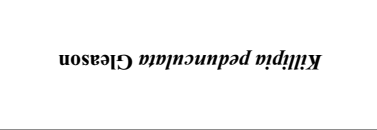 & 苞 & $\begin{array}{l}\text { 惢 } \\
\text { 离 }\end{array}$ & 0 & 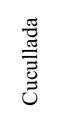 & $\stackrel{\circ}{\dddot{2}}$ & 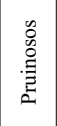 & 总 & in & $\$$ & مิ & $\mathrm{F}$ & के & $\vec{s}$ & 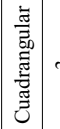 & m & 岂 & 8 & 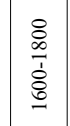 & 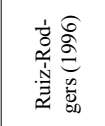 \\
\hline 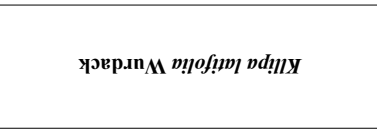 & 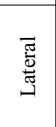 & $\begin{array}{l}\frac{5}{0} \\
\text { 总 }\end{array}$ & 0 & 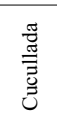 & 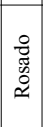 & 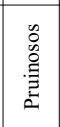 & \begin{tabular}{|l}
$\dot{0}$ \\
$\dot{\vec{d}}$ \\
0 \\
0 \\
0 \\
0 \\
$\dot{1}$
\end{tabular} & in & 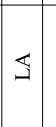 & ิิ & 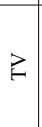 & in & $\therefore$ & 总 & m & 罟 & 8 & 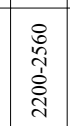 & 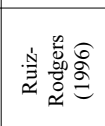 \\
\hline צрвр.пи & 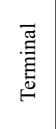 & 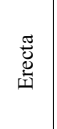 & 0 & 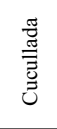 & 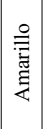 & 善 & 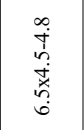 & in & $\Xi$ & ลิ & F & $\vec{n}$ & $\bar{\infty}$ & 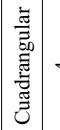 & ॰ & 崖 & 8 & 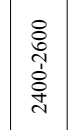 & 曾 \\
\hline 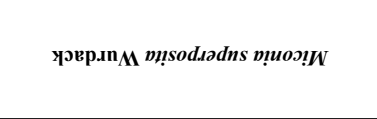 & 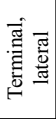 & 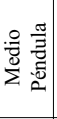 & 0 & $\begin{array}{l}\frac{\tilde{g}}{\tilde{m}} \\
\frac{\overline{\bar{z}}}{\mathrm{z}} \\
\end{array}$ & 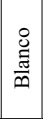 & $\therefore$ & 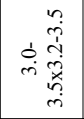 & in & $\$$ & ิิ & $\rho$ & के & $\therefore$ & 总 & m & 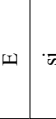 & 8 & 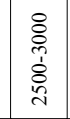 & 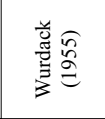 \\
\hline 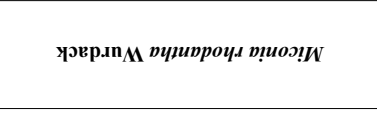 & 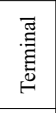 & 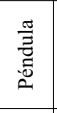 & 0 & 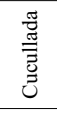 & 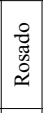 & 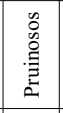 & 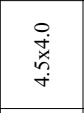 & n & $\$$ & $\hat{\mathrm{a}}$ & ? & $\bar{n}$ & $\vec{n}$ & 总 & m & 罗 & 8 & 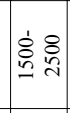 & 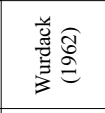 \\
\hline 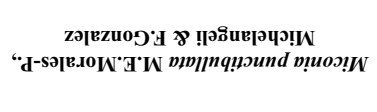 & 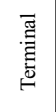 & 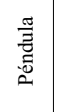 & 0 & $\begin{array}{l}\frac{\tilde{g}}{\tilde{m}} \\
\frac{\bar{J}}{\bar{J}} \\
\bar{J}\end{array}$ & 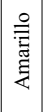 & 亭 & 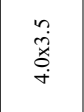 & n & $\leftrightarrows$ & $\hat{\mathrm{O}}$ & F & $\vec{n}$ & $\vec{n}$ & 总 & a & 崩的 & 8 & 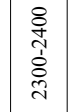 & 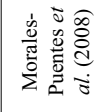 \\
\hline уовр..п & 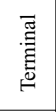 & $\begin{array}{l}\text { 䕸 } \\
\text { 离 }\end{array}$ & 吾 & 总 & 善 & 总 & 妾 & in & 0 & ลิ & 亏 & क & $\vec{n}$ & 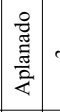 & 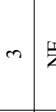 & 岸 & 8 & 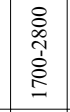 & 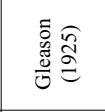 \\
\hline 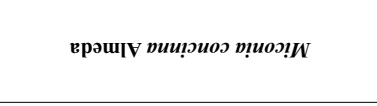 & 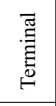 & 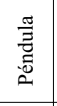 & 0 & 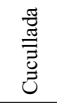 & 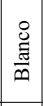 & 总 & 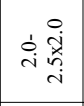 & in & $\$$ & ิิ & F & क & $\vec{n}$ & 总: & 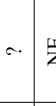 & $\begin{array}{ll}\mathbf{z} \\
\end{array}$ & 0 & 㝘: & 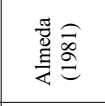 \\
\hline урер..и & 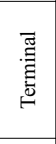 & 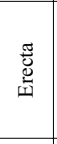 & 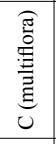 & 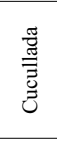 & $\stackrel{\circ}{\dddot{2}}$ & 惫 & 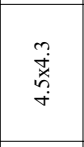 & in & 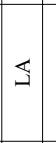 & ิิ & F & $\bar{n}$ & $\therefore$ & 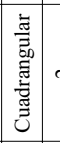 & a & \begin{tabular}{|l|l|l}
$\mathbf{z}$ \\
\end{tabular} & 8 & 亲 & 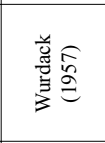 \\
\hline 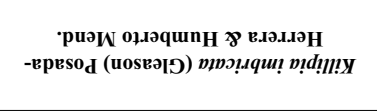 & 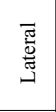 & 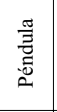 & 0 & 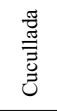 & $\begin{array}{l}8 \\
\frac{\delta}{\tilde{m}} \\
\frac{m}{m}\end{array}$ & \begin{tabular}{|l} 
总 \\
o. \\
言 \\
至
\end{tabular} & 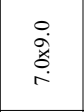 & in & 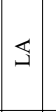 & ิิ & F & क & $\vec{x}$ & 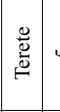 & in & $\begin{array}{ll}\mathbf{z} \\
\end{array}$ & 8 & 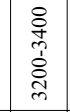 & 言 \\
\hline иохе & 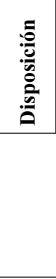 & 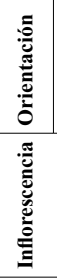 & $\stackrel{\circ}{\Xi}$ & 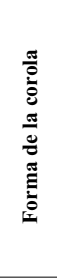 & 递 & 㺃 & 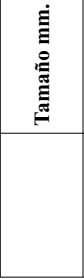 & 这 & 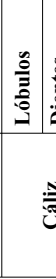 & 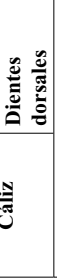 & 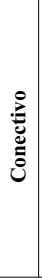 & 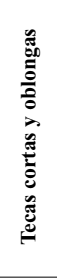 & 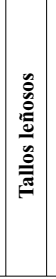 & $\mid$ & 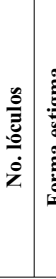 & | & 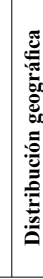 & 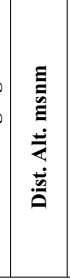 & 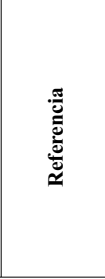 \\
\hline
\end{tabular}




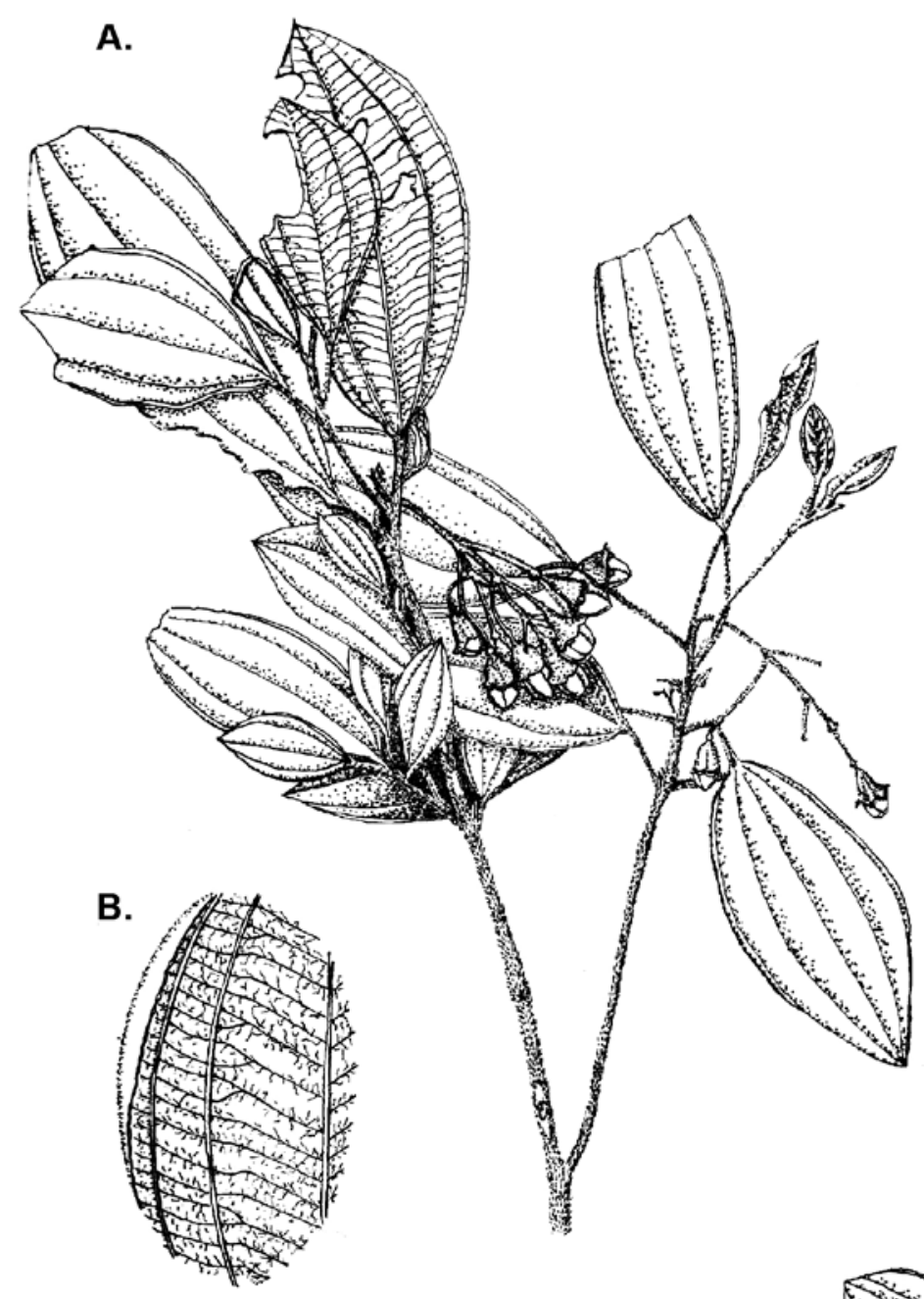

C.

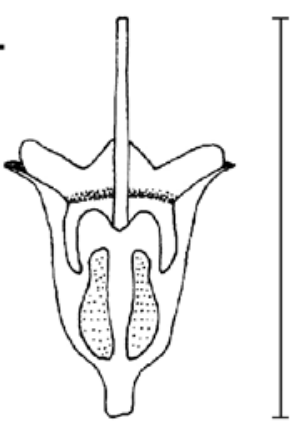

$13,0 \mathrm{~mm}$

D.

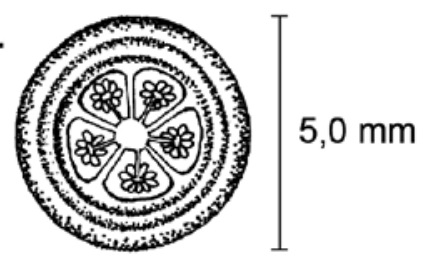

E.

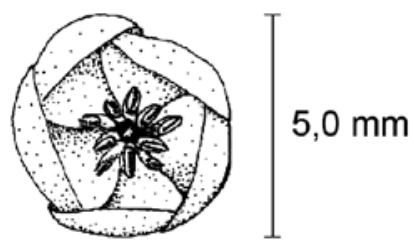

G.
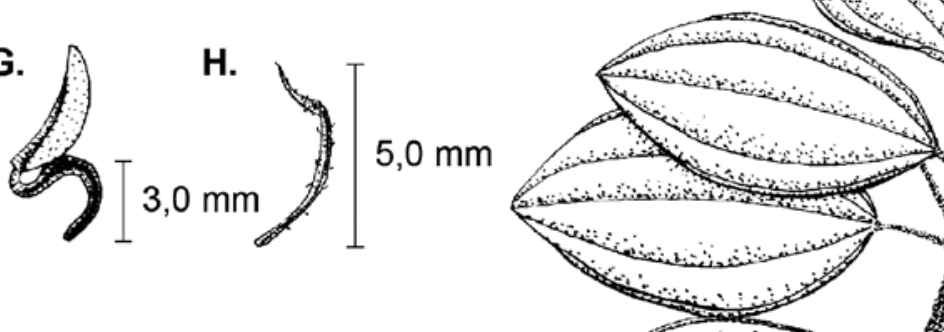

F.

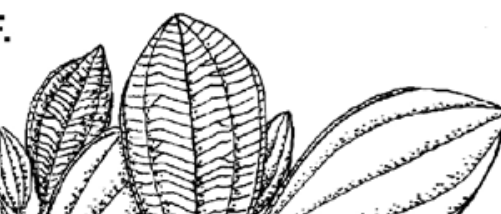

I.

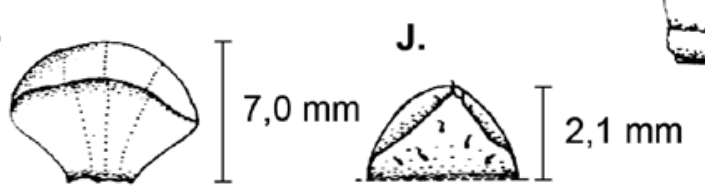

K.

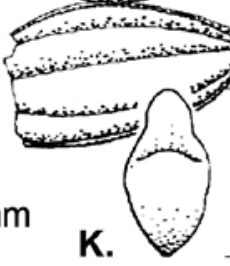

2
5
5
$\frac{1}{3}$
$\frac{3}{4}$
$\frac{5}{5}$

Figura 2. Killipia imbricata. A. Hábito con botones florales B. Detalle del envés. C. Corte longitudinal del hipanto. D. Corte trasversal del hipanto E. Detalle de la corola. F. Habito con frutos. G. Estambre. H. Bractéola. I. Pétalo. J. Dientes del cáliz. K. Placenta. Ilustración de las partes basada en D. Sanín 4269 (FMB); A y F, basados en J. M. Posada 127 (FAUC, COL). 

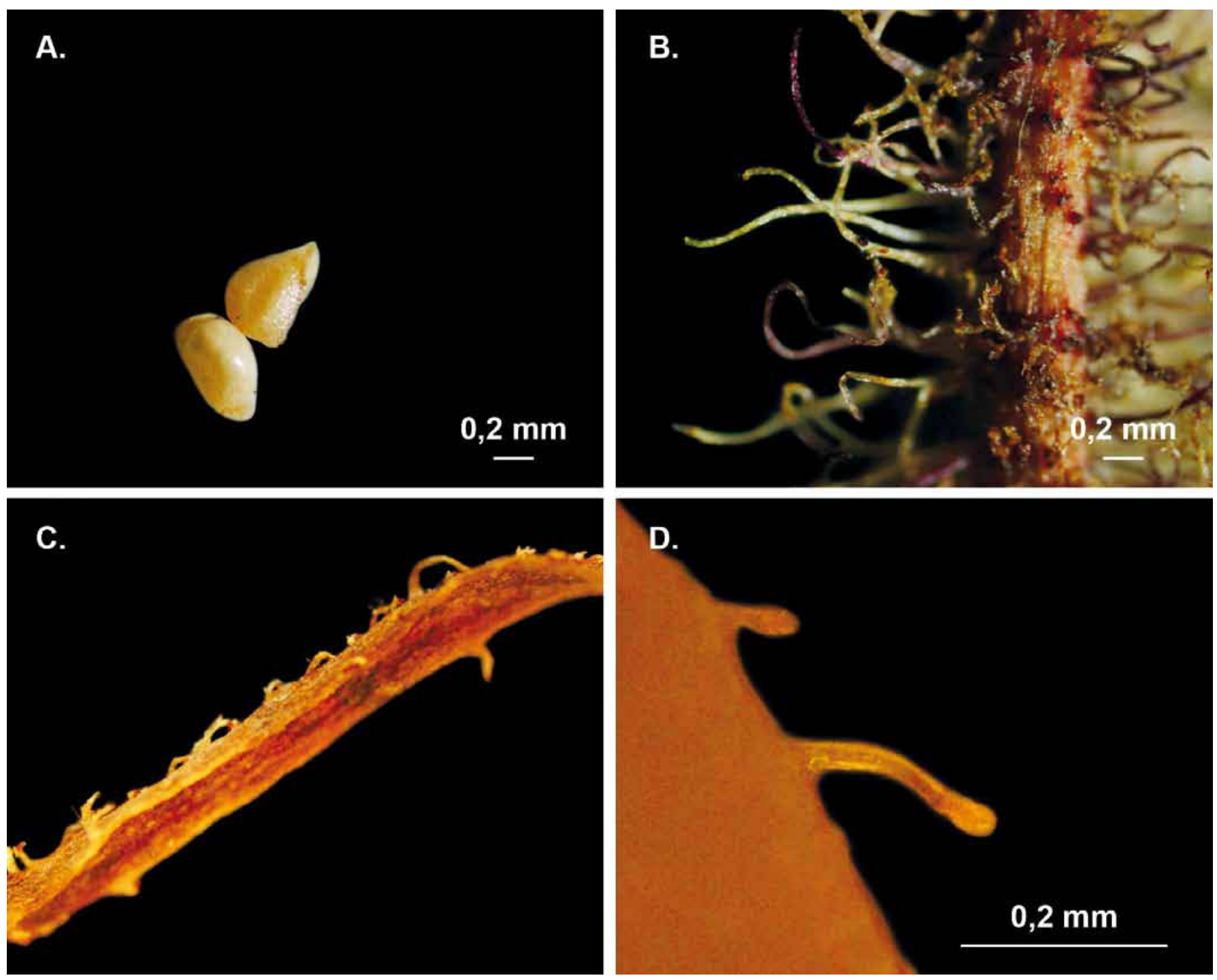

Figura 3. Killipia imbricata. A. Semillas. B. Tricomas de las hojas. C. Bractéolas. D. Tricomas del pistilo. A, basado en J.M. Posada 127 (FAUC, COL); B, C, D, basados en D. Sanín 4269 (FMB).

un retículo conspicuo; toda la nervadura con cubierta densa de tricomas filamentosos de $1.3-1.5 \mathrm{~mm}$, transparentes excepto en los nervios principales que son de color púrpura (café claro en material seco). Inflorescencia de apariencia terminal pero se desarrolla en la axila de una rama atrofiada, paniculada-corimbosa, péndula, de 3-5 cm, con 8-12 flores; eje central con 3-4 nudos de ramificación, primer internodo terete y de $0,9-1,6 \mathrm{~cm}$; paracladios basales $1-1,7 \mathrm{~cm}$, con 1 nivel de ramificación; ápice de las ramillas dicasiales; brácteas caedizas $5-7 \mathrm{~mm}$. Flores pediceladas, 5 meras, diplostemonas; pedicelo 3-6 mm. Hipanto campanulado, de 4-5 x 3-4mm, paredes de $0,5 \mathrm{~mm}$ de grosor, externamente con cubiertas como las ramas pero menos densa, tricomas de $0,16-$
0,24 $\mathrm{mm}$; torus $0,9 \mathrm{~mm}$ de grosor. Cáliz lobulado; lóbulos anchamente redondeados, de 3,0 x 1,7 mm, membranáceos y glabros; dientes externos anchamente triangulares y apiculados, de $2,1 \mathrm{~mm}$ de largo, externamente con cubierta como el hipanto. Corola levemente acopada (cucullada); pétalos 7x9 $\mathrm{mm}$, anchamente obovados, apicalmente inflexos, blancos, externamente pruinosos, internamente glabros. Estambres isomórficos, dispuestos simétricamente y en circulo alrededor del estilo; anteras y conectivo de color amarillo claro; filamentos de $3 \mathrm{~mm}$ longitud y $0,8 \mathrm{~mm}$ de ancho cerca a la base; conectivo minutamente tuberculado en la parte basaldorsal, estos pequeños apéndices de 0,6 x 0,6 mm; tecas de $4 \mathrm{~mm}$, oblongas, con poro apical o levemente dorsal. Estilo 
columnar y recto, de 7,6 $\mathrm{mm}$, base de $0,6 \mathrm{~mm}$ de diámetro, con tricomas glandulares esparcidos cerca de la base de $0,2-$ $0,3 \mathrm{~mm}$; estigma no expandido en material seco, levemente expandido en material fresco, de $0,6-0,7 \mathrm{~mm}$ de diámetro. Ovario 5 locular, parcialmente fusionado en la base $3 \mathrm{~mm}$ al hipanto, ovoide, de 5,0 x 2,5 mm; ápice con dientes anchos sobresalientes $0,7 \mathrm{~mm}$; placentas de $1,8 \times 0,8 \mathrm{~mm}$, con rudimentos en todas las superficies. Fruto baya, de 2,5-3,5 mm de longitud sin el pedicelo, diámetro 2,5-3mm. Semilla obovada o ovoide, testa de color café claro, rafe conspicua, más que la testa y levemente rugosa, $0,5-0,6 \times 0,33-0,35 \mathrm{~mm}$.

Distribución y hábitat: Especie hasta ahora restringida al Parque Nacional Natural Tatamá, en el departamento de Risaralda, municipio de Santuario, en zonas de bosque Altoandino entre los 3200 y $3400 \mathrm{~m}$ de altitud.

Fenología: Las tres únicas colecciones conocidas poseen flores y provienen de los meses de septiembre y enero, que coinciden con los periodos lluviosos y secos en la zona de referencia (Rangel-Ch et al., 2005).

Notas taxonómicas: Esta especie se puede diferenciar de otras especies de Killipia por crecer en zonas de bosque Altoandino, por poseer tallo terete, por la cubierta densa de tricomas filamentosos color purpura en fresco y café claro cuando seco en las partes vegetativas e inflorescencia, la inflorescencia péndula, la corola de color blanco, el ápice del cáliz con lóbulos redondeados membranáceos con dientes dorsales anchamente triangulares y los estambres de color amarillo, con el conectivo dorsal basal levemente tuberculado.

Estado de Conservación: Por ser endémica de zonas de subpáramo-paramo con un área de 13.875ha (Parques Nacionales Naturales de Colombia, 2007) y restringida a una localidad, puede ser catalogada como Vulnerable (dependiente de un área de conservación).

Ejemplares revisados: COLOMBIA. Risaralda: Santuario, PNN Tatamá, sector entre El Fracaso-Valle de Las Mirlas El Fracaso, $3400 \mathrm{~m}, 5^{\circ} 07^{\prime} 21.58^{\prime \prime} \mathrm{N}, 7^{\circ} 02^{\prime} 45.63^{\prime} \mathrm{W}, 15$ ene 2011, fl, D, Sanín 4269, (FMB); fr, J.M, Posada 127, (FAUC, COL).

\section{Agradecimientos}

Los autores agradecen al personal del Parque Nacional Natural Tatamá por permitir el acceso, a su director Hugo Ballesteros y a Rosa Restrepo (funcionaria del parque) por su apoyo y colaboración. Al Instituto de Investigaciones en Estratigrafía-IIES, en especial al profesor Andres Pardo por el apoyo para realizar la expedicion. Al investigador David
Sanín, por la logistica, su compañía en campo, facilicitacion de sus colecciones y amable colaboracion al facilitar el acceso a las instalaciones del instituto. A Julio Andrés Sierra Giraldo y Luis Fernando Coca por su apoyo en campo. A Melissa Zuluaga por la edición de las imágenes.

\section{Referencias}

Almeda, F. 1981. New and reconsidered species of Miconia (Melastomataceae) from Costa Rica and Panama. Proc. Calif. Acad. Sci. 42(10): 303-314.

Gleason, H. 1925. Studies on the flora of northern South America-VII. Bull. Torrey Bot. Club 52(7): 373-388.

Goldenberg, R. 2000. O gênero Miconia Ruiz \& Pav. Melastomataceae. I. Listagens analítica. II. Revisão taxonômica da seção Hypoxanthus (Rich. ex DC.) Hook. Tesis de Doctorado. Universidad de Campinas, Campinas, Brasil.

Judd, W. S. 1986. Taxonomic studies in the Miconieae (Melastomataceae) I. Variation in inflorescence position. Brittonia 38: 150-161.

Judd, W. S. 1989. Taxonomic studies in the Miconieae (Melastomataceae) III. Cladistic analysis of axillary-flowered taxa. An. Missouri Bot. Gard 76: 476-495.

Lozano-Contreras, G. \& N. Ruiz-Rodgers. 1995. Nuevas especies de $\mathrm{Sa}$ graea (Melastomataceae). Mutisia 75: 1-11.

Mendoza, H. \& B. Ramírez. 2007. Lista preliminar de especies de Melastomataceae y Memecylaceae para Colombia. Instituto de Investigación de Recursos Biológicos Alexander von Humboldt; Universidad del Cauca. Bogotá D.C. Colombia. http://www.humboldt.org.co/ humboldt/mostrarpagina.php? codpage $=2000$

Michelangeli, F., D. Penneys, J. Giza, D. Soltis, M.H. Hils \& J.D. Skean Jr. 2004. A preliminary phylogeny of the tribe Miconieae (Melastomataceae) based on nrITS sequence data and its implications on inflorescence position. Taxon 53(2): 279-290.

Morales-Puentes, M. E., F. Michelangeli, F. González. 2008. Una nueva especie de Miconia (Melastomataceae: Miconieae) de Colombia. Brittonia 60(3): 228-234.

Parques Nacionales Naturales de Colombia. 2007. Plan de manejo 20072011, Parque Nacional Natural Tatamá. Dirección Territorial Noroccidente. Medellín, Antioquia. (Resumen ejecutivo).

Rangel Ch, J.O., M. AguilarP, A. Flores. 2005. Clima en el área del transecto Tatamá. Pags. 47-103. En: Van der Hammen, Th., J. O. Rangel Ch. \& A. M. Cleef (eds.). Studies on Tropical Andean Ecosystems Vol. 6. La Cordillera Occidental Colombiana. Transecto Tatamá. J. Cramer, Berlin, Stuttgart. (9)

Ruiz-Rodgers, N. 1996. Estudio sistemático del género Killipia (Melastomataceae: Miconieae) en Colombia. Trabajo de grado Magister en Biología - Línea Sistemática, Universidad Nacional de Colombia. Bogotá.

Ruiz-Rodgers, N. 1997. Una nueva especie del genero Killipia (Melastomataceae). Caldasia 19(1-2): 175-177.

Wurdack, J. J. 1955. Certamen Melastomataceis-II. Phytologia 5(4): 130.

Wurdack, J. J. 1957. Certamen Melastomataceis-IV. Brittonia 9(2): 101109.

Wurdack, J. J. 1962. Certamen Melastomataceis VII. Phytologia 8(4):168-17

Recibido: 6 de mayo de 2013

Aceptado para su publicación: 4 de junio de 2013 